\title{
Collagen Extraction from Malaysian Cultured Catfish (Hybrid Clarias Sp.): Kinetics and Optimization of Extraction Conditions Using Response Surface Methodology
}

\author{
Peck Loo Kiew and Mashitah Mat Don \\ School of Chemical Engineering, Universiti Sains Malaysia, 14300 Nibong Tebal, Malaysia \\ Correspondence should be addressed to Mashitah Mat Don, chmashitah@eng.usm.my
}

Received 4 October 2012; Accepted 28 November 2012

Academic Editors: K. T. Valsaraj and P. P. Wieczorek

Copyright ( $) 2012$ P. L. Kiew and M. Mat Don. This is an open access article distributed under the Creative Commons Attribution License, which permits unrestricted use, distribution, and reproduction in any medium, provided the original work is properly cited.

\begin{abstract}
A central composite design (CCD) was used for the experimental design and results analysis to obtain the optimal processing parameters (acetic acid concentration, liquid to solid ratio, and stirring speed) for the extraction of pepsin soluble collagen (PSC) from muscles of cultured hybrid catfish of Clarias sp. (Clarias gariepinus $\times$ C. macrocephalus). Statistical analysis showed that the linear and quadratic terms of these three independent variables had significant effects on the yield of PSC. There was also an interaction between the ratio of liquid to solid and the stirring speed in affecting the extraction efficiency. Optimal conditions for a higher yield of PSC were an acetic acid concentration of $0.67 \mathrm{M}$, a liquid to solid ratio of $24.65 \mathrm{ml} / \mathrm{g}$, and the stirring speed of $423.64 \mathrm{rpm}$. The verification of the optimization showed that the percentage error differences between the experimental and predicted values were in the range of $0.22-4.42 \%$. The experimental values agreed with the predicted values, indicating an excellent fit of the model used and the success of the response surface methodology in modeling the extraction of PSC from the muscles of catfish. The experimental results were also fitted to the power law model and it was proven to be appropriate in describing the kinetics of collagen extraction process.
\end{abstract}

\section{Introduction}

For decades, collagen has been exploited in medical applications owing to its extraordinary biocompatibility and safety, particularly the ability to form fibers with extra strength and high stability by self-aggregation in the drug delivery system [1]. It serves as the most popular precursor of gelatin, which is widely applied to commercial products [2]. In fact, the application of collagen also diversifies in food, cosmetics, pharmaceutical, and cell culture industries. It is gradually emerging as another popular food additive in ham, sausage, and other food for mouth feel improvement, and is recently regarded as an effective edible condiment for skin care [3]. Consumption of collagen has increased with the development of new industrial applications, leading to an increasing demand for this biomaterial nowadays. Sources of collagen in the industries are primarily derived from cattle hides, beef bones, and pork skin. However, health-conscious consumers are opposing the utilization of collagen extracted from these land animals due to health concerns. Besides the risks of transferring bovine spongiform encephalopathy (BSE), foot-and-mouth disease, and avian flu, porcine-derived collagen is not permitted to be used by Muslims and Jews for religious reasons, while collagens extracted from bovine sources are prohibited for Sikhs and Hindus [4]. Therefore, raw materials from fishery products have attracted attention as alternative sources of consumerfriendly collagen [5].

Locally known as Keli in Malaysia, cultured hybrid catfish of Clarias sp. (Clarias gariepinus $\times$ C. macrocephalus) is one of the most popular freshwater fishes accepted by consumers contributing by its abundances and cheaper price as compared to other cultured fishes. Catfish is a good source of protein with a considerable amount of collagen exists in the musclesand skins [6]. In our preliminary study, it was found that this hybrid Clarias catfish contained the highest amount 
of pepsin soluble collagen (PSC) among other Malaysian freshwater fishes such as red tilapia (Oreochromis niloticus), black tilapia (Oreochromis mossambicus), pangasius catfish (Pangasius sutchi), Sultan fish (Leptobarbus hoevenii), and labyrinth fish (Trichogaster trichopterus). Demand for freshwater fishes in Malaysia is only meant for daily consumption so far, resulting in lower commercial value in contrast to deep sea species. Therefore, the extraction of collagen from catfish aids in boosting up the commercial value of these cheaply abundant natural resources besides providing a new approach in finding alternative sources of safe collagen for industrial uses. Collagen is a thermosensitive compound and the extraction process is normally extracted under the condition that is lower than the room temperature $[1,7]$. It is very crucial to understand the effects of extraction conditions in order to obtain the highest yield of collagen under the most optimum operating conditions.

The efficiency of the collagen extraction process from the muscle of Clarias sp. can be influenced by many process factors such as acid concentration, extraction time, stirring speed, and liquid to solid ratio [1, 7]. Under such circumstances where multiple variables may affect the extraction yield, response surface methodology (RSM) is an effective technique in optimizing the process [8]. RSM is a mathematical modeling technique that relates independent and dependent variables and subsequently generates regression equations that represent the interrelations between the input parameters and output properties [4]. In short, the principles and applications of RSM involve three steps: (1) experimental design where the independent variables and the corresponding experimental levels are set using statistical experimental designs such as the central composite design (CCD) or Box-Behnken design (BBD); (2) response surface modeling through regression analysis; and (3) process optimization using the response surface model [8]. Few reports that are available in the literature demonstrated that RSM is an effective tool for investigating the optimum collagen extraction conditions from the skin of yellowfin tuna [2], grass carp [1], scales of yellowfin tuna [3], and swim bladder of grass carp [7]. Nevertheless, publications on collagen extraction with response surface methodology are still very limited. To our best knowledge, the optimization of extraction of collagen from muscles of fishes using RSM has not been reported yet.

The aim of this work was to determine the optimum conditions for the extraction of collagen from the muscles of hybrid Clarias sp. using acetic acid as the extracting solvent. It was conducted by analyzing the influence of extraction conditions on the yield obtained at the end of the extraction process using the response surface methodology (RSM). Acetic acid concentration, liquid to solid ratio, and stirring speed were the optimized variables. RSM was applied to find out the best extraction conditions in order to maximize the yield of extracted collagen, with the minimization of the energy cost of the process. Additionally, kinetics of the extraction process under selected conditions was also investigated. Mathematical modeling of solid-liquid extraction processes is an important engineering tool in the design process in order to reduce energy, time, and chemical reagents consumption [9]. In our previous study, the power law model was found to be the best model capable of predicting the collagen extraction data for various species of Malaysian freshwater fishes [10]. Therefore, the influence of each process factor on the model parameters was also assessed in this paper.

\section{Materials and Methods}

2.1. Materials. Cultured catfish (hybrid of C. gariepinus $\times$ C. macrocephalus) were purchased from a local wet market in Parit Buntar, Perak, Malaysia. Upon arrival at the laboratory, the fishes were killed, and dissected, deboned and the muscles were cleaned of adhering tissues before being cut into small pieces. Skin was manually removed by using a sharp knife. The muscles were then washed with distilled water and kept frozen at $-20^{\circ} \mathrm{C}$ prior to the collagen extraction.

2.2. Chemicals. Commercial pepsin from porcine gastric mucosa, sodium hydroxide, and acetic acid was purchased from Merck Sdn. Bhd. (Malaysia). All other chemicals used were of analytical grade.

\subsection{Isolation of Muscle Type I Collagen}

2.3.1. Extraction of Pepsin Soluble Collagen (PSC). All procedures were performed as previously described by Kimura et al. [11] and Wang et al. [1] with slight modifications. The extraction processes were carried out at $4^{\circ} \mathrm{C}$. To remove noncollagenous proteins, the muscles were mixed with 10 volumes $(\mathrm{v} / \mathrm{w})$ of $0.1 \mathrm{M} \mathrm{NaOH}$ and stirred for 5 to $6 \mathrm{hr}$. The sample was then washed thoroughly with excessive distilled water until the $\mathrm{pH}$ was neutral or slightly basic. The treated fish muscles were subjected to collagen extraction by aqueous acetic acid. Acetic acid concentration, liquid to solid ratio, and stirring speed were chosen as independent variables with different levels. The extraction process was carried out with the aid of pepsin digestion with an enzyme/substrate ratio of 1:40 for $24 \mathrm{hr}$ to extract pepsin soluble collagen. The concentration of collagen extracted was measured using Lowry's modified method as reported by Komsa-Penkova et al. [12]. After centrifugation at $3,840 \times \mathrm{g}$ for $15 \mathrm{~min}$, soluble collagen solution was obtained from the supernatant. The collagen was precipitated by adding $\mathrm{NaCl}$ to a final concentration of $2.0 \mathrm{M}$ in the presence of $0.05 \mathrm{M}$ Tris$\mathrm{HCl}$ buffer ( $\mathrm{pH}$ 7.2). Resulting sediment was collected by centrifugating at $3,840 \times \mathrm{g}$ for $20 \mathrm{~min}$. The purified collagen was redissolved in minimal amount of $0.5 \mathrm{M}$ acetic acid, and dialyzed against $0.1 \mathrm{M}$ acetic acid, followed by distilled water and lyophilized. The freeze-dried product was designated as pepsin soluble collagen (PSC).

2.4. Collagen Yield Measurement. The yield of pepsin soluble collagen from muscle of Clarias sp. was calculated using (1) as proposed by Li et al. [13]:

$$
Y=\frac{V \times C}{W}
$$


where $Y$ is the yield of collagen in $\mathrm{mg} / \mathrm{g}, V$ is the volume of extracted collagen solution in $\mathrm{mL}, C$ is the concentration of the same solution measured using spectrophotometer in $\mathrm{mg} / \mathrm{mL}$, and $W$ is the wet weight of catfish muscle in $g$.

2.5. Experimental Design and Analysis of Data. Response surface methodology was employed for experimental design, data analysis, and model building with the aid of the software Design Expert (Version 6.0.6, Stat-Ease Inc., Minneapolis, Minnesota, USA). Central composite design (CCD) with three variables was used to determine the response pattern and then to establish a model. According to the CCD, the total number of experimental combinations is $2^{k}+2 k+n_{0}$, where $k$ is the number of independent variables and $n_{0}$ is the number of repetitions of the experiments at the centre point. For statistical calculation, the experimental variables $X_{i}$ have been coded as $x_{i}$ according to the following transformation equation:

$$
x_{i}=\frac{X_{i}-X_{0}}{\partial X},
$$

where $x_{i}$ is the dimensionless coded value of the variable $X_{i}, X_{0}$ is the value of $X_{i}$ at the center point, and $\partial X$ is the step change [7]. In this study, the central composite design with three factors and three levels, including six replicates at the center point, was used for fitting a second-order response surface. Three independent variables used in this work were acetic acid concentration $\left(X_{1}\right)$, liquid to solid ratio $\left(X_{2}\right)$, and stirring speed $\left(X_{3}\right)$, while the dependent variable was the yield of PSC. The ranges and center point values of all independent variables were based on the results of preliminary experiments. The yield of PSC was analyzed by multiple regressions to fit into the following polynomial equation:

$$
Y=\beta_{0}+\sum_{i=1}^{3} \beta_{i} X_{i}+\sum_{i=1}^{3} \beta_{i i} X_{i}^{2}+\sum_{i=1}^{2} \sum_{j=i+1}^{3} \beta_{i j} X_{i} X_{j},
$$

where $Y$ is a dependent variable (yield of PSC expressed in milligram of collagen per gram of fish muscle), $\beta_{0}$ is intercept, $\beta_{i}, \beta_{i i}, \beta_{i j}$ are regression coefficients, and $X_{i}$ indicates the linear terms, $X_{i}^{2}$ for the quadratic terms for a single variable, and $X_{i} X_{j}$ for the interaction terms [14]. Six replicates at the central point of the designed model were used to estimate the pure error sum of squares. In order to evaluate the effect of the process variables, 20 experiments were performed in random order to cover all combinations of the factor levels in the experimental design. All experiments were done in triplicate and data presented were mean values of the triplicates.

2.6. Extraction Kinetic Model. Publication available on the kinetic model of collagen extraction from fish muscles is still scarce in the literature. Kinetic models can be divided into physical and empirical. According to Kitanović et al. [15], empirical models described the mathematic variations of the amount of extracted compound in either the raw material or liquid extract with time. They are normally simpler as compared to the physical models but are appropriate for engineering purposes. In this paper, a two-parametric kinetic model known as the power law was validated based on the selected process conditions. The authors have previously compared the suitability of few empirical kinetic models which included the power law, parabolic diffusion, and Weibull's and Elovich's models in governing the kinetics of collagen extraction from muscles of few selected Malaysian freshwater fishes. The power law model has successfully provided the best fit in describing the muscles collagen extraction kinetics from all fishes among all other models. Therefore, it was chosen in this study. Kinetic modeling of the extraction by applying the power law was based on the following assumptions:

(i) fish muscles were isotropic and of equal size;

(ii) distribution of collagen within the fish muscles was uniform and varied only with time;

(iii) net diffusion occurred only towards the external surface of fish muscles;

(iv) diffusion coefficient of collagen was a constant.

The power law model, which was similar to the Freundlich type, was applied widely in the diffusion process of an active agent through nonswelling devices [15]. It could be applied as follows:

$$
\mathrm{y}=B t^{n},
$$

where $y$ is the yield of collagen $(\mathrm{mg} / \mathrm{g}), B$ refers to the constant incorporating the characteristics of the carrieractive agent system, $t$ is the time in minutes, and $n$ is the diffusional exponent, an indicative of transport mechanism. In literature, $n$ was less than 1 for extraction from plant or vegetal materials. The constants for this model must be estimated using a regression analysis. In the linearized form, the equation was transformed into

$$
\ln y=n \ln t+\ln B .
$$

The influence of each process factor on the kinetic model parameter was assessed by analyzing the linear regression equations.

2.7. Statistical Methods. Experimental data was analyzed by multiple regressions to fit the quadratic equation to all independent variables. Analysis of variance (ANOVA) was performed to evaluate significant differences between independent variables. Relationships between the response and independent variables were visualized using surface response and contour plots of the fitted quadratic regression equations generated by Design Expert (Version 6.0.6, Stat-Ease Inc., Minneapolis, Minnesota, USA). In order to validate the kinetic model, the kinetic parameter values obtained from different extraction process conditions were used to simulate the profiles for the proposed power law model. The profiles from simulation of the experimental data and models were then evaluated using the linear correlation coefficient, $R^{2}$, 
and the root mean square deviation (RMSD) computed as follows [16]:

$$
\mathrm{RMSD}=\sqrt{\frac{1}{N} \sum_{i=1}^{N}\left(y_{\text {exp }}-y_{\text {calc }}\right)^{2}} .
$$

\section{Results and Discussion}

3.1. Statistical Analysis and Model Fitting. All 20 experimental points were evaluated and the collagen extraction yield results based on the factorial design are shown in Table 1. Response surface optimization is favorable over the conventional single parameter optimization in that it saves time, space, and raw materials [8]. A maximum PSC extraction yield of $222.76 \pm 9.37 \mathrm{mg} / \mathrm{g}$ was recorded under the experimental conditions of acetic acid concentration $0.5 \mathrm{M}$, liquid to solid ratio $25 \mathrm{~mL} / \mathrm{g}$, and stirring speed of $400 \mathrm{rpm}$. By applying multiple regression analysis on the experimental data, the predicted response variable and the independent variables were related in terms of coded values by the following quadratic equation:

$$
\begin{aligned}
Y= & 210.86+44.11 X_{1}+20.14 X_{2}+21.60 X_{3}-50.32 X_{1}^{2} \\
& -0.92 X_{2}^{2}-26.32 X_{3}^{2}+6.61 X_{1} X_{2}+2.16 X_{1} X_{3} \\
& -12.25 X_{2} X_{3},
\end{aligned}
$$

where $Y=$ yield of extracted collagen $(\mathrm{mg} / \mathrm{g}) ; X_{1}=$ acetic acid concentration $(\mathrm{M}) ; X_{2}=$ liquid to solid ratio $(\mathrm{mL} / \mathrm{g})$; and $X_{3}=$ stirring speed (rpm). The coefficients with one factor represent the effect of that particular factor, while the coefficients with two factors and those with second-order terms represent the interaction between the two factors and the quadratic effect, respectively. The positive sign of the terms indicates the synergistic effect, while the negative sign is indicating an antagonistic effect [17]. ANOVA was used to evaluate the significance of the coefficients of the model. The regression coefficient values of equation are listed in Table 3.

The $P$ values were used as a tool to check the significance of each coefficient, subsequently indicating the pattern of the interactions between the variables. Zhang et al. [8] stated that for any of the terms in a particular model, a large regression coefficient and a small $P$ value are implying a more significant effect on the respective response variables. This indicates that the smaller is the values of $P$, the more significant is the corresponding coefficient. From Table 2, it can be seen that the linear coefficients $\left(X_{1}, X_{2}, X_{3}\right)$ and the quadratic term coefficients $\left(X_{1}, X_{3}\right)$ were significant, with very small $P$ values $(P<0.05)$. The interaction between the coefficient $X_{2}$ and $X_{3}$, namely, the liquid to solid ratio and stirring speed, was also another significant effect in the proposed model with the $P$ value at 0.0175 .

According to Zhang et al. [7] and Wang et al. [1], exploration and optimization of a fitted response surface might produce poor or misleading results unless the model exhibits a good fit, which makes the checking of the model adequacy essential. The $P$ value of the model was less than 0.0001 (Table 3). Meanwhile, the lack of fit value of the model
TABLE 1: Response surface CCD and results for yield of PSC extraction.

\begin{tabular}{ccccc}
\hline & $\begin{array}{c}X_{1}, \\
\text { acetic acid } \\
\text { concentration } \\
(\mathrm{M})\end{array}$ & $\begin{array}{c}X_{2}, \\
\text { liquid to solid } \\
\text { ratio } \\
(\mathrm{mL} / \mathrm{g})\end{array}$ & $\begin{array}{c}X_{3}, \\
\text { stirring } \\
\text { speed } \\
(\mathrm{rpm})\end{array}$ & $\begin{array}{c}Y, \\
\text { yield of } \\
\text { extraction PSC } \\
(\mathrm{mg} / \mathrm{g})\end{array}$ \\
\hline 1 & $-1(0.1)$ & $-1(20)$ & $-1(300)$ & $51.04 \pm 0.85$ \\
2 & $1(0.9)$ & $-1(20)$ & $-1(300)$ & $116.54 \pm 3.35$ \\
3 & $-1(0.1)$ & $1(30)$ & $-1(300)$ & $50.93 \pm 0.35$ \\
4 & $1(0.9)$ & $1(30)$ & $-1(300)$ & $151.67 \pm 5.37$ \\
5 & $-1(0.1)$ & $-1(20)$ & $1(500)$ & $100.51 \pm 2.17$ \\
6 & $1(0.9)$ & $-1(20)$ & $1(500)$ & $183.45 \pm 3.34$ \\
7 & $-1(0.1)$ & $1(30)$ & $1(500)$ & $60.20 \pm 1.51$ \\
8 & $1(0.9)$ & $1(30)$ & $1(500)$ & $160.82 \pm 4.87$ \\
9 & $-1(0.1)$ & $0(25)$ & $0(400)$ & $110.50 \pm 4.55$ \\
10 & $1(0.9)$ & $0(25)$ & $0(400)$ & $201.82 \pm 6.78$ \\
11 & $0(0.5)$ & $-1(20)$ & $0(400)$ & $190.91 \pm 3.38$ \\
12 & $0(0.5)$ & $1(30)$ & $0(400)$ & $170.22 \pm 1.66$ \\
13 & $0(0.5)$ & $0(25)$ & $-1(300)$ & $139.55 \pm 0.54$ \\
14 & $0(0.5)$ & $0(25)$ & $1(500)$ & $220.77 \pm 9.88$ \\
15 & $0(0.5)$ & $0(25)$ & $0(400)$ & $217.56 \pm 7.56$ \\
16 & $0(0.5)$ & $0(25)$ & $0(400)$ & $203.67 \pm 5.73$ \\
17 & $0(0.5)$ & $0(25)$ & $0(400)$ & $212.45 \pm 10.51$ \\
18 & $0(0.5)$ & $0(25)$ & $0(400)$ & $206.45 \pm 5.55$ \\
19 & $0(0.5)$ & $0(25)$ & $0(400)$ & $222.76 \pm 9.37$ \\
20 & $0(0.5)$ & $0(25)$ & $0(400)$ & $219.78 \pm 8.46$ \\
\hline & & & &
\end{tabular}

was 0.0722 which was not significant. These two values confirmed that the model fitness was good. In addition, a lower value of the coefficient of variation (CV) at 7.09\% which is lower than $10 \%$ indicated a better precision and reliability of the experiments [18]. CV was frequently used as an indication of the degree of precision with which the treatments were compared. The higher the value of $\mathrm{CV}$, the lower the reliability of the experiment is [7]. As for the adequate precision value, which is a measure of the signal (response) to noise (deviation) ratio, a ratio greater than four is desirable [8]. In this study, the ratio was found to be 21.58 , indicating an adequate signal and therefore the model is significant for the extraction process. Another important analysis by ANOVA was the checking of the precision of a particular model by the determination of correlation coefficient $\left(R^{2}\right)$. It is defined as the ratio of the explained variation to the total variation and is a measurement of the degree of fitness [18]. A regression model having a $R^{2}$ value higher than 0.9 or approaches unity is considered to have a very high correlation and the model can fit well with experimental data. Likewise, a small value of $R^{2}$ implies a poor relevance of the dependent variables in the model $[1,7]$. Here, the value of $R^{2}(0.9733)$ for (7) indicates a close agreement between the experimental results and theoretical values predicted by the model equation. It is also proven that the proposed regression model is able to define the true behavior of the collagen extraction system well. 
TABLE 2: Estimated coefficients of the fitted quadratic polynomial equation for different responses.

\begin{tabular}{|c|c|c|c|c|c|}
\hline Parameter & Regression coefficient & Standard error & $F$ value & $P$ value & Indication \\
\hline \multicolumn{6}{|l|}{ Linear } \\
\hline$X_{1}$ & 44.11 & 3.86 & 130.85 & $<0.0001$ & Significant \\
\hline$X_{2}$ & 20.14 & 3.86 & 27.27 & 0.0004 & Significant \\
\hline$X_{3}$ & 21.60 & 3.86 & 31.38 & 0.0002 & Significant \\
\hline \multicolumn{6}{|l|}{ Quadratic } \\
\hline$X_{1}^{2}$ & -50.32 & 7.35 & 46.82 & $<0.0001$ & Significant \\
\hline$X_{2}^{2}$ & -0.92 & 7.35 & 0.016 & 0.9034 & Not significant \\
\hline$X_{3}^{2}$ & -26.32 & 7.35 & 12.81 & 0.0050 & Significant \\
\hline \multicolumn{6}{|l|}{ Interaction } \\
\hline$X_{1} X_{2}$ & 6.61 & 4.31 & 2.35 & 0.1560 & Not significant \\
\hline$X_{1} X_{3}$ & 2.16 & 4.31 & 0.25 & 0.6266 & Not significant \\
\hline$X_{2} X_{3}$ & -12.25 & 4.31 & 8.07 & 0.0175 & Significant \\
\hline
\end{tabular}

TABle 3: Analysis of variance (ANOVA) for the response surface quadratic model of the yield of PSC from muscles of Clarias sp.

\begin{tabular}{lccccc}
\hline Source & $\begin{array}{c}\text { Degrees of } \\
\text { freedom }\end{array}$ & $\begin{array}{c}\text { Sum of } \\
\text { squares }\end{array}$ & $\begin{array}{c}\text { Mean } \\
\text { square }\end{array}$ & $F$ value & $P$ value \\
\hline Model & 9 & 54266.64 & 6029.63 & 40.54 & $<0.0001$ \\
Residual & 10 & 1487.16 & 148.72 & & \\
Lack of fit & 5 & 1198.49 & 239.70 & 4.15 & 0.0722 \\
Pure error & 5 & 288.67 & 57.73 & & \\
\hline Total & 19 & 55753.80 & & & \\
$R^{2}=0.9733$; Dependent mean $=172.08 ;$ Adj. $R^{2}=0.9493 ; \mathrm{CV}=7.09$.
\end{tabular}

3.2. Comparison of Observed and Predicted PSV Yield. A regression model provides the ability to predict future observations on the response $Y$ (PSC yield) corresponding to particular values of the variables [7]. However, there is the possibility that a model fits well in the region of the original data will no longer fit well beyond the region. Therefore, extrapolating beyond the region containing the original observations in predicting new response at a given point must be done cautiously. Figure 1 shows the experimental PSC yield (the response) versus those predicted from the empirical model (7). It clearly illustrates that the predicted data of the response from the model is in agreement with the observed ones in the range of the operating variables.

3.3. Optimization of the PSC Extraction Conditions. Graphical representations of the regression model, known as the response surfaces and contour plots, were obtained with the aid of Design Expert software version 6.0.6. The results of the PSC extraction yield as influenced by acetic acid concentration, liquid to solid ratio, and stirring speed are illustrated in Figures 2-4. Response surface methodology plays a key role in identifying the optimum values of the independent variables efficiently, under which the response variable can achieve a maximum response [8]. In the response surface and contour plot, the extraction yield of PSC was obtained along with two continuous variables, whilst the remaining variable was held constant at the respective zero level (center value

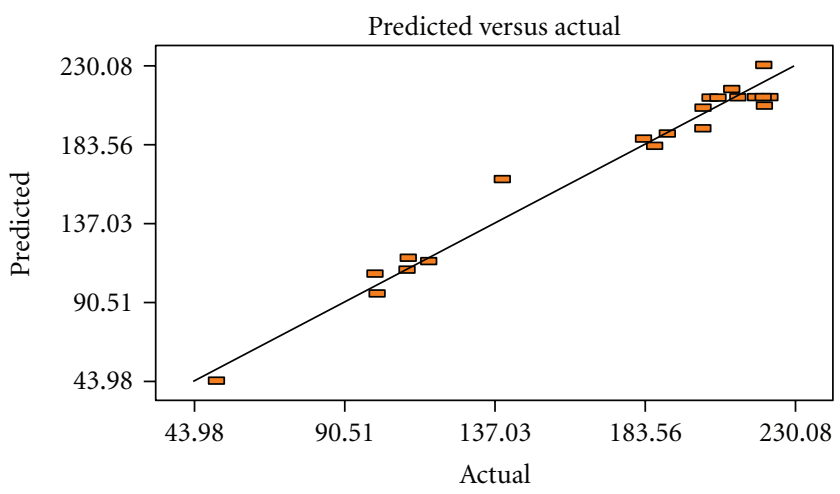

FIGURE 1: Predicted PSC yield versus the experimental yield under different extraction conditions.

of the testing ranges) $[2,19]$. Shapes of the corresponding contour plots indicate whether mutual interactions between the independent variables are significant or not [7]. Elliptical contours are obtained when there is a perfect interaction between the independent variables $[20,21]$, and the maximum predicted value indicated by the surface is confined to the smallest ellipse in the contour diagram [8]. From the $3 \mathrm{D}$ response surface plots, optimal values of the independent variables could be observed, and the interaction between each independent variable's pair could be easily understood.

The effects of acetic acid concentration and liquid to solid ratio on the yield of PSC from muscles of Clarias sp. are shown in Figure 2. The yield of PSC increased with the increase of acetic acid concentration to a certain value (approximately $0.5 \mathrm{M}$ ), and thereafter decreased. A similar trend was observed in Figure 3 where the interaction between acetic acid concentration and the stirring speed is shown. The yield of PSC increased with the increase of acetic acid concentration to $0.5 \mathrm{M}$. However, a reverse trend was observed beyond this concentration. Difference in the yield obtained through different concentrations of acetic acid employed was probably due to a different solubility of collagen in the acidic extracting medium. According to a few reports, $\mathrm{pH}$ value of the extraction bulk was completely 


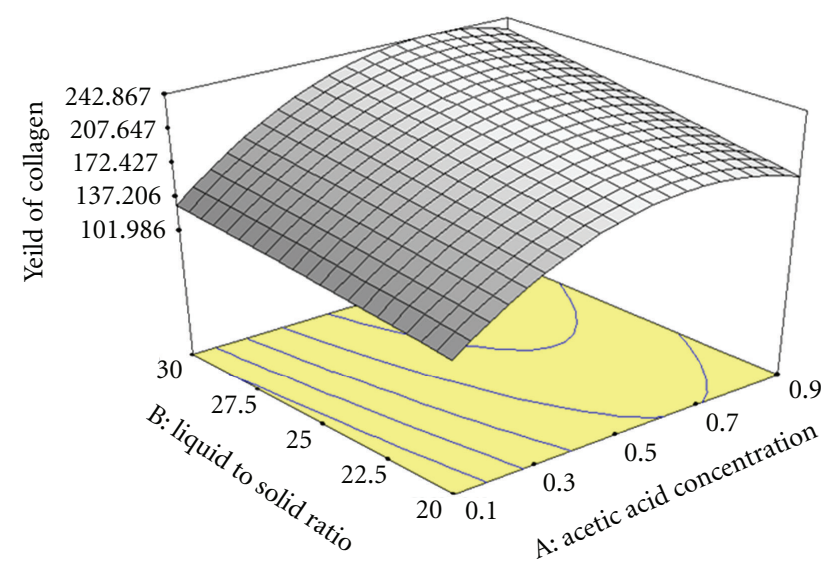

Figure 2: Response surface plot showing the effects of acetic acid concentration and liquid to solid ratio on the yield of PSC. The stirring speed was constant at $400 \mathrm{rpm}$.

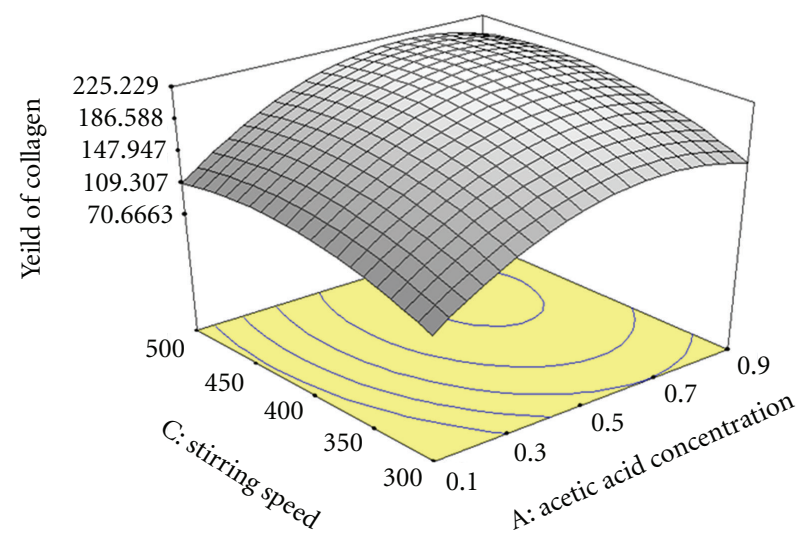

Figure 3: Response surface plot showing the effects of acetic acid concentration and stirring speed on the yield of PSC. The liquid to solid ratio was constant at $25 \mathrm{~mL} / \mathrm{g}$.

dependent on the concentration of acid used. Hence, the modification of the electrostatic interaction and structure of proteins might occur along the changes in acid concentration since $\mathrm{pH}$ value was in charge of the charge density of protein [22]. In fact, Wang et al. [23] recently stipulated that more positively charged amine groups of collagen were resulted at the $\mathrm{pH}$ when the concentration of acetic acid used was at $0.5 \mathrm{M}$, leading to the highest yield among the studied concentrations. Denaturation of collagen at extremely low $\mathrm{pH}$ value $(\mathrm{pH}<2)$ however was also another possibility that lower yield was observed when acetic acid with concentration more than $0.5 \mathrm{M}$ was utilized.

A positive relationship was found between liquid to solid ratio and the yield of PSC (Figures 2 and 4). Solvent to material ratio is an important variable affecting the efficiency of extraction. An increasing acetic acid to muscle ratio could lead to a higher yield of PSC. The ratio was varied from 20 to $30 \mathrm{~mL} / \mathrm{g}$, but when it was raised to more than $25 \mathrm{~mL} / \mathrm{g}$, the improvement in the yield of PSC was no longer significant. Even though this phenomenon was not so obvious in

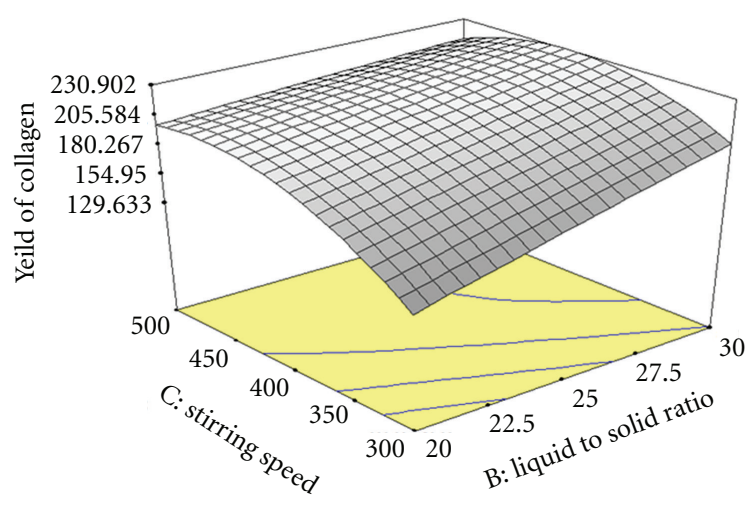

Figure 4: Response surface plot showing the effects of liquid to solid ratio and stirring speed on the yield of PSC. The acetic acid concentration was constant at $0.5 \mathrm{M}$.

Figure 4, higher solvent to material ratio increases the concentration gradient and diffusion rate of collagen particles from the fish muscles into acetic acid, thus enhancing the efficiency of extraction process [23]. Nevertheless using a large amount of solvent is not cost effective due to a higher operating cost of solvent and waste handling at the end of the extraction process. Similar trend was also observed for the effect of stirring speed on the amount of PSC extracted along the process. The amount of PSC extracted increased significantly $(P<0.05)$ with the elevation of stirring speed from $300 \mathrm{rpm}$ to $400 \mathrm{rpm}$ (Figures 3 and 4). This could be due to the mass transfer between the fish muscles and acetic acid which was greatly enhanced with increasing stirring speed. Greater solubility of muscles in acetic acid was achieved, resulting in greater driving force for collagen particles to diffuse from the fish muscles into the medium. Further increase of the speed after $400 \mathrm{rpm}$ however did not result in a significant improvement of the yield.

Among the three extraction parameters studied, acetic acid concentration was the most significant factor affecting the yield of PSC in this extraction process, followed by stirring speed, and the liquid to solid ratio. This was proven by the regression coefficients significance of the quadratic model (Table 2) and gradient of slope in the 3D response surface plot (Figures 2-4).

3.4. Validation of Predictive Model. By prediction with computing program, the optimal conditions to obtain the highest yield of PSC were identified as follows: an acetic acid concentration of $0.67 \mathrm{M}$, the liquid to solid ratio of $24.65 \mathrm{~mL} / \mathrm{g}$, and the stirring speed of $423.64 \mathrm{rpm}$. In order to check on the adequacy of the predictive model, 5 sets of experiments were repeated at the suggested optimum conditions in order to experimentally obtain the maximum yield of PSC from the muscles of Clarias sp. As shown in Table 4, the percentage error differences between the experimental and predicted values were in the range of 0.22 $4.42 \%$. Since the differences between the experimental and predicted response were always less than $5 \%$, the validity of the model was verified. 
TABLE 4: Validation of the data and constructed model.

\begin{tabular}{lccc}
\hline & $\begin{array}{c}\text { Experimental yield } \\
(\mathrm{mg} / \mathrm{g})\end{array}$ & $\begin{array}{c}\text { Predicted yield } \\
(\mathrm{mg} / \mathrm{g})\end{array}$ & $\begin{array}{c}\text { Error } \\
(\%)\end{array}$ \\
\hline Trial 1 & $228.71 \pm 7.33$ & $224.62 \pm 0.00$ & 1.82 \\
Trial 2 & $225.12 \pm 4.29$ & $224.62 \pm 0.00$ & 0.22 \\
Trial 3 & $214.69 \pm 6.42$ & $224.62 \pm 0.00$ & 4.42 \\
Trial 4 & $223.29 \pm 9.18$ & $224.62 \pm 0.00$ & 0.59 \\
Trial 5 & $220.33 \pm 6.87$ & $224.62 \pm 0.00$ & 1.91 \\
\hline
\end{tabular}

3.5. Effects of Extraction Conditions on Kinetic Model Parameters. Extraction of collagen from muscles of freshwater fishes could be divided into two periods: (i) fast washing action in the very beginning and (ii) a slow diffusion controlled extraction in the last period. Washing was characterized by the rapid increase in the yield of collagen in early beginning of the extraction process, followed by slow extraction approximately after the first 16 hours of the process. Correlation between amounts of collagen extracted by pepsin digestion from fish muscles related to time was found to be appropriately represented by the power law, parabolic diffusion, and Elovich's equations in our previous study, suggesting that a diffusive mechanism governs collagen release from the fish muscles [10]. In fact, the power law was selected as the best empirical model for the muscle collagen extraction from freshwater fishes based on the highest linear correlation value, $R^{2}$, and the lowest value of root mean square deviation, RMSD. Therefore, effects of different process factors which can significantly affect the extraction process such as the concentration of acetic acid, liquid to solid ratio, and stirring speed towards the parameters of the power law were investigated in this study. Figure 5 illustrates the profiles of experimental and simulated data for the extraction of collagen from Clarias sp. using the power law model at different process conditions.

The predicted results gave a relatively good agreement to the experimental data, with the $R^{2}$ values above 0.9 . In fact, the $R^{2}$ values were high for all process conditions, ranging $0.910<R^{2}<0.995$. This showed that the proposed power low model was adequate in describing both the fast washing action and slow diffusion of extraction process for PSC. However, many examples exist where the $R^{2}$ is closed enough to one but the model is still not appropriate. Hence the root mean square deviation (RMSD) was used with the $R^{2}$ for the confirmation of adequacy of the power model. A model with small RMSD represents the data more accurately than the models with larger RMSD [15]. Table 5 shows the calculated model parameters and the corresponding statistical correlation values. It turned out that regardless of the difference in process conditions, the individual average value of the RMSD was lower than $10 \%$ for all the conditions.

The influence of each process factor and their interactions on the kinetic model parameters was assessed by analyzing the linear regression equations. As it can be seen from Table 5, acetic acid concentration, liquid to solid ratio, and stirring speed affected parameters of the power law model significantly. Empirical models can be useful for initial rate estimations, which are important for extraction processes [24]. For collagen extraction process in this study, the coefficient $n$ of the power law model refers to the extraction rate constant, and the initial extraction rate is represented by the term $B$. The above analysis led to the following findings.

(a) When the acetic acid concentration increased from 0.1 to $0.5 \mathrm{M}$, the initial extraction rate increased significantly. The increase of $B$ might be related to the increasing initial solubility of collagen (PSC) from fish muscles into the acidic solvent. As stated earlier, $\mathrm{pH}$ value of the extraction bulk was completely dependent on the concentration of the acid used. Therefore, initial solubility of collagen was greatly affected by the concentration of acetic acid. With further increase of concentration to 0.7 and $0.9 \mathrm{M}$, the initial extraction rate reduced drastically.

(b) With increasing liquid to solid ratio from 10 to $30 \mathrm{~mL} / \mathrm{g}$, an increase in $n$ and decrease in $B$ was observed. This was probably due to lower initial solubility of collagen in the solvent with the increasing amount of solvent used. Even though there were claims that at higher solvent to material ratio, the concentration gradient and diffusion rate of solute into solvent could be enhanced [23], but in this case, the decrease in the initial extraction rate could be due to the lesser contact between the fish muscles with the acidic solvent. This was because stirring speed of the extraction process was set constant even though the amount of solvent used was increased. As the result, no improvement in the extraction rate yet decrease in the mass transfer between the collagen particles and solvent was observed.

(c) At constant acetic acid concentration and liquid to solid ratio used, higher stirring speed could improve the initial extraction rate. This was obvious that the value of $B$ was significantly increased with the increasing stirring speed from 200 to $500 \mathrm{rpm}$. Mass transfer is limited by diffusion, thus the more efficient the stirring is, the better the mixing between solvent and raw material, the shorter the equilibrium time, and the higher the amount of analyte would be extracted in preequilibrium conditions [25]. Therefore, initial solubility of collagen in the acetic acid directly affected the initial collagen extraction rate in this study.

\section{Conclusions}

Statistical optimization of process conditions using the central composite design (CCD) appeared to be an effective tool for the extraction process of pepsin soluble collagen (PSC) from the muscles of Malaysian cultured catfish (Clarias sp.). Each of the three independent variables (acetic acid concentration, liquid to solid ratio, and stirring speed) showed a significant effect in the yield of extracted PSC. The mathematical model gave an $R^{2}$ of 0.9733 and a $P$ 
TABLE 5: Summary of model parameters and statistical correlation values for each studied process condition.

\begin{tabular}{|c|c|c|c|c|}
\hline \multirow{2}{*}{ Process conditions } & \multicolumn{2}{|c|}{ Kinetic model parameter } & \multicolumn{2}{|c|}{ Statistical correlation values } \\
\hline & $n$ & $B\left(\min ^{-n}\right)$ & $R^{2}$ & RMSD (\%) \\
\hline \multicolumn{5}{|c|}{ Acetic acid concentration (M) } \\
\hline 0.1 & 0.460 & 1.730 & 0.990 & 2.847 \\
\hline 0.5 & 0.176 & 40.977 & 0.943 & 2.621 \\
\hline 0.7 & 0.286 & 18.690 & 0.958 & 3.588 \\
\hline 0.9 & 0.387 & 7.838 & 0.995 & 1.611 \\
\hline \multicolumn{5}{|c|}{ Liquid to solid ratio (mL/g) } \\
\hline 10 & 0.176 & 40.977 & 0.943 & 2.621 \\
\hline 20 & 0.305 & 21.264 & 0.978 & 2.758 \\
\hline 25 & 0.378 & 14.835 & 0.910 & 7.208 \\
\hline 30 & 0.489 & 6.347 & 0.942 & 7.295 \\
\hline \multicolumn{5}{|l|}{ Stirring speed (rpm) } \\
\hline 200 & 0.681 & 0.879 & 0.971 & 7.000 \\
\hline 300 & 0.692 & 1.091 & 0.990 & 4.011 \\
\hline 400 & 0.378 & 14.835 & 0.910 & 7.208 \\
\hline 500 & 0.27 & 29.548 & 0.959 & 3.358 \\
\hline
\end{tabular}

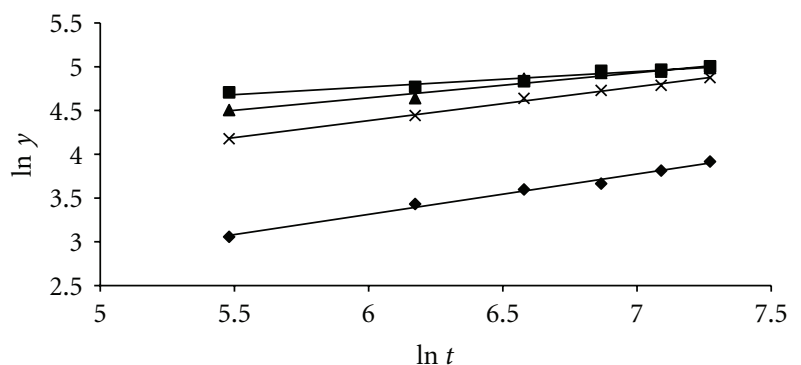

- $0.1 \mathrm{M}$

- $0.5 \mathrm{M}$

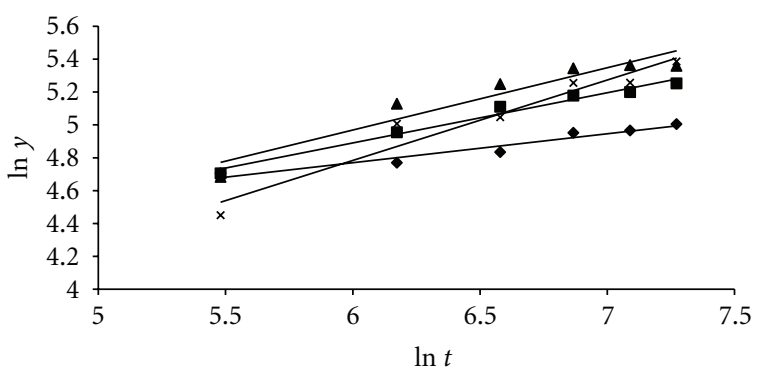

- $10 \mathrm{~mL} / \mathrm{g}$

- $20 \mathrm{~mL} / \mathrm{g}$

- $25 \mathrm{~mL} / \mathrm{g}$

$\times 30 \mathrm{~mL} / \mathrm{g}$

(a)

(b)

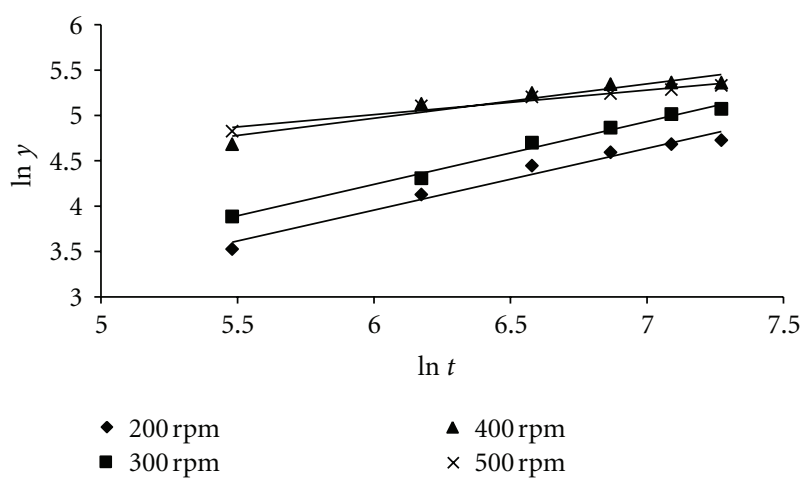

(c)

FIgURE 5: Comparison of experimental (symbols) and fitted (line) data for the extraction of collagen from Clarias sp. based on the linearized form of the power law model: (a) effects of acetic acid concentration; (b) effects of liquid to solid ratio; and (c) effects of stirring speed.

value of less than 0.0001 , which implied a good agreement between the predicted values and the actual values of the yield of PSC, thus confirmed a good generalization of the mathematical model. The optimal conditions to obtain maximum yield of PSC were identified as follows: $0.67 \mathrm{M}$ of acetic acid concentration, $24.65 \mathrm{~mL} / \mathrm{g}$ of liquid to solid ratio, and stirring speed at $423.64 \mathrm{rpm}$. Under these optimized conditions, the experimental PSC extraction yield agreed closely with the predicted yield of $224.62 \mathrm{mg} / \mathrm{g}$. The power law model was also found to be successfully representing the PSC extraction process under all studied process conditions, with high linear correlation coefficient and relatively low 
RMSD, suggesting that the release of collagen from fish muscles was governed by a diffusive mechanism. In order to achieve certain values of the extraction rate, the process factors have to be identified and controlled accordingly. The results in this work were helpful for the extraction of PSC from muscles of catfish which has a very low commercial value in the market. However, further research on the structural characteristics of collagen is important to understand its physical and chemical properties.

\section{Acknowledgments}

The authors gratefully acknowledge the USM Fellowship provided by the Universiti Sains Malaysia (USM) and Exploratory Research Grant Scheme (account no: 203/ PJKIMIA/6730068) from the Ministry of Higher Education (MOHE), Malaysia, to support this paper. The authors would also like to extend their gratitude to Madam Hajah Haslawati Baharuddin and Tuan Haji Rosly bin Hassan of the Malaysia Freshwater Fisheries Research Center (FFRC) for valuable advices in species identification of the cultured catfish.

\section{References}

[1] L. Wang, B. Yang, X. Du, Y. Yang, and J. Liu, “Optimization of conditions for extraction of acid-soluble collagen from grass carp (Ctenopharyngodon idella) by response surface methodology," Innovative Food Science and Emerging Technologies, vol. 9, no. 4, pp. 604-607, 2008.

[2] J. W. Woo, S. J. Yu, S. M. Cho, Y. B. Lee, and S. B. Kim, "Extraction optimization and properties of collagen from yellowfin tuna (Thunnus albacares) dorsal skin," Food Hydrocolloids, vol. 22, no. 5, pp. 879-887, 2008.

[3] Y. Han, J. R. Ahn, J. W. Woo et al., "Processing optimization and physicochemical characteristics of collagen from scales of yellowfin tune (Thunnus albacares)," Fisheries and Aquatic Sciences, vol. 13, pp. 102-111, 2010.

[4] H. Yang, Y. Wang, M. Jiang, J. H. Oh, J. Herring, and P. Zhou, "2-Step optimization of the extraction and subsequent physical properties of channel catfish (Ictalurus punctatus) skin gelatin," Journal of Food Science, vol. 72, no. 4, pp. C188C195, 2007.

[5] H. J. Kim, M. S. Yoon, K. H. Park, J. H. Shin, M. S. Heu, and J. S. Kim, "Processing optimization of gelatin from rockfish skin based on yield," Fisheries and Aquatic Science, vol. 13, no. 1, pp. 1-11, 2010.

[6] P. Sivakumar, R. Arichandran, L. Suguna, M. Mariappan, and G. Chandrakasan, "The composition and characteristics of skin and muscle collagens from a freshwater catfish grown in biologically treated tannery effluent water," Journal of Fish Biology, vol. 56, no. 4, pp. 999-1012, 2000.

[7] B. Zhang, Y. Chen, X. Wei, M. Li, and M. Wang, "Optimization of conditions for collagen extraction from the swim bladders of grass carp (ctenopharyngodon idella) by response surface methodology," International Journal of Food Engineering, vol. 6, no. 3, pp. 1-18, 2010.

[8] L. L. Zhang, M. Xu, Y. M. Wang, D. M. Wu, and J. H. Chen, "Optimizing ultrasonic ellagic acid extraction conditions from infructescence of platycarya strobilacea using response surface methodology," Molecules, vol. 15, no. 11, pp. 7923-7932, 2010.
[9] N. Piwowarska and J. González-Alvarez, "Extraction of antioxidants from forestry biomass: kinetics and optimization of extraction conditions," Biomass and Bioenergy, vol. 43, pp. 4251, 2012.

[10] P. L. Kiew and M. Mat Do, "Screening and empirical kinetic models of collagenextraction from selected Malaysian freshwater fish," Journal of Food Process Engineering. In press.

[11] S. Kimura, X. P. Zhu, R. Matsui, M. Shijoh, and S. Takamizawa, "Characterization of fish muscle type I collagen," Journal of Food Science, vol. 53, pp. 1315-1318, 1988.

[12] R. Komsa-Penkova, R. Spirova, and B. Bechev, "Modification of Lowry's method for collagen concentration measurement," Journal of Biochemical and Biophysical Methods, vol. 32, no. 1, pp. 33-43, 1996.

[13] D. Li, C. Mu, S. Cai, and W. Lin, "Ultrasonic irradiation in the enzymatic extraction of collagen," Ultrasonics Sonochemistry, vol. 16, no. 5, pp. 605-609, 2009.

[14] S. Zeng, X. Yan, W. Cao, P. Hong, C. Zhang, and L. Li, “Optimisation of extraction conditions and characteristics of skin gelatin from Nile tilapia (Oreochromis niloticus)," International Journal of Food Science and Technology, vol. 45, no. 9, pp. 18071813, 2010.

[15] S. Kitanović, D. Milenović, and V. B. Veljković, "Empirical kinetic models for the resinoid extraction from aerial parts of St. John's wort (Hypericum perforatum L.)," Biochemical Engineering Journal, vol. 41, pp. 1-11, 2008.

[16] S. Jokić, D. Velić, M. Bilić, A. Bucić-kojić, and M. Pianinić, "Modelling of the process of solid-liquid extraction of total polyphenols from soybeans," Czech Journal of Food Science, vol. 28, pp. 206-212, 2010.

[17] L. Rodrigues, J. Teixeira, R. Oliveira, and H. C. Van Der Mei, "Response surface optimization of the medium components for the production of biosurfactants by probiotic bacteria," Process Biochemistry, vol. 41, no. 1, pp. 1-10, 2006.

[18] A. Nath and P. K. Chattopadhyay, "Optimization of oven toasting for improving crispness and other quality attributes of ready to eat potato-soy snack using response surface methodology," Journal of Food Engineering, vol. 80, no. 4, pp. 1282-1292, 2007.

[19] D. B. Uma, C. W. Ho, and W. M. Wan Aida, "Optimization of extraction parameters of total phenolic compounds from henna (Lawsonia inermis) leaves," Sains Malaysiana, vol. 39, no. 1, pp. 119-128, 2010.

[20] R. V. Muralidhar, R. R. Chirumamila, R. Marchant, and P. Nigam, "A response surface approach for the comparison of lipase production by Candida cylindracea using two different carbon sources," Biochemical Engineering Journal, vol. 9, no. 1, pp. 17-23, 2001.

[21] C. Chang, P. Cen, and X. Ma, "Levulinic acid production from wheat straw," Bioresource Technology, vol. 98, pp. 1448-1453, 2007.

[22] M. Verheul, S. P. F. M. Roefs, and K. G. De Kruif, "Kinetics of heat-induced aggregation of $\beta$-lactoglobulin," Journal of Agricultural and Food Chemistry, vol. 46, no. 3, pp. 896-903, 1998.

[23] L. Wang, B. Yang, and X. Du, "Extraction of acid-soluble collagen from grass carp (ctenopharyngodon idella) skin," Journal of Food Process Engineering, vol. 32, no. 5, pp. 743-751, 2009.

[24] P. Bansal, M. Hall, M. J. Realff, J. H. Lee, and A. S. Bommarius, "Modeling cellulase kinetics on lignocellulosic substrates," Biotechnology Advances, vol. 27, no. 6, pp. 833-848, 2009. 
[25] R. Sanja, V. Dajana, and P. Janusz, "Solid phase microextraction," in Handbook of Sample Preparation, J. Pawliszyn and L. L. Heather, Eds., p. 94, John Wiley \& Sons, New York, NY, USA, 2010. 

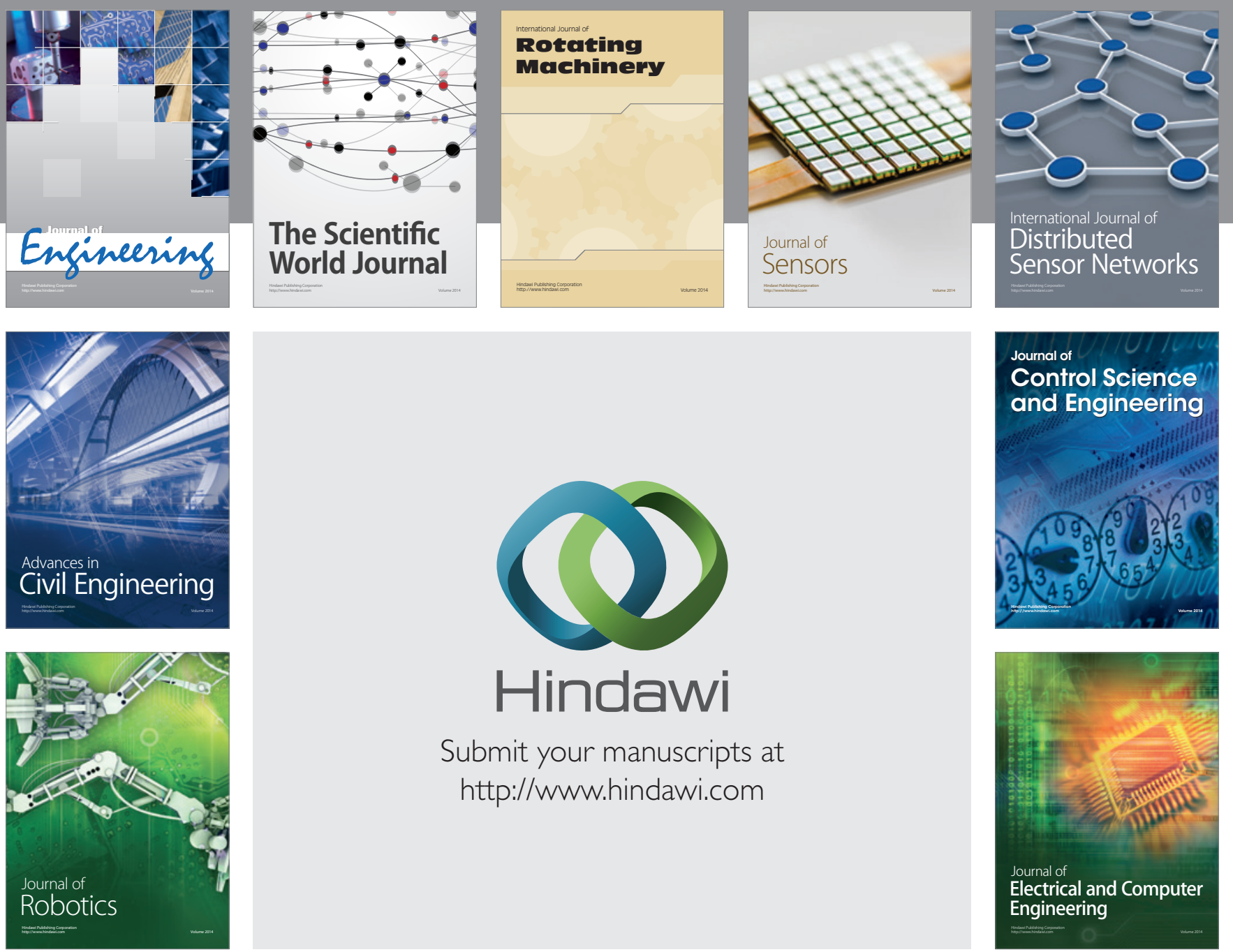

Submit your manuscripts at

http://www.hindawi.com
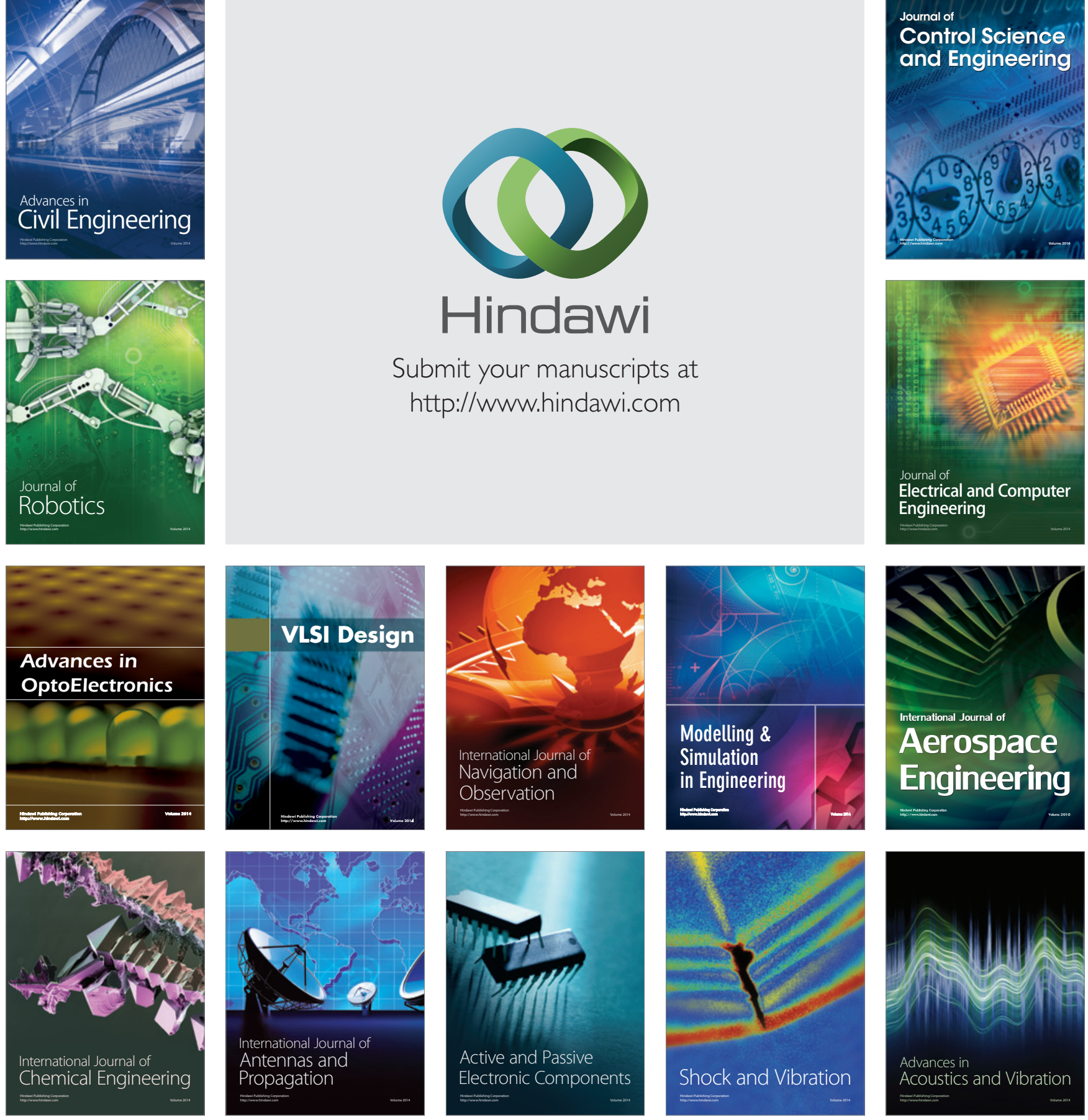